\title{
IMPRONTA ANDINA ENTRE LOS KAMASKOS DEL WENULEUFU*
}

\author{
AN ANDEAN IMPRINT AMONG THE KAMASKOS \\ OF WENULEUFU
}

\section{RODRIGO MOULIAN**, PABLO ESPINOZA*}

\section{RESUMEN}

El presente artículo expone la recurrencia de rasgos panandinos en las prácticas y representaciones sagradas de los miembros de las congregaciones religiosas mapuche williche en la cuenca del Río Bueno, conocidos como kamaskos. A partir del estudio comparativo de tramas simbólicas mostramos el correlato de los paradigmas cosmovisionarios centro y sur andinos en tópicos como las formas de ancestralización, las representaciones escatológicas, la concepción del paisaje y de los elementos de la naturaleza. El carácter sistemático de estas correlaciones, que constituye un indicador de vínculos de filiación cultural, configura lo que denominamos la impronta andina en la cuenca del Río Bueno o Wenuleufu.

Palabras clave: Williche, andinidad, cosmovisión, cotradición.

\section{ABSTRACT}

This paper presents the recurrence of pan-Andean features in practices and sacred representations of members of Mapuche williche religious congregations in the Río Bueno river basin, known as kamaskos. From the comparative study of symbolic frames, we show the correlation of central and southern Andean worldview paradigms in top-

* Artículo elaborado en el marco del proyecto Fondecyt 1120139, "La impronta andina el sistema religioso cosmovisionario mapuche williche" y DID S 2012-2, "La alimentación de las almas, la muerte y las transformaciones del espíritu en el espacio centro y sur andino".

** Doctor en Antropología, académico de la Universidad Austral de Chile, Valdivia, Chile. Correo electrónico: rmoulian@hotmal.com

*** Magíster en Comunicación, académico de la Universidad de Tarapacá, Arica, Chile. Correo electrónico: pespinozac@uta.cl 
ics as ancestralization, eschatological representations, conception of the landscape and the elements of nature. The systematic character of these correlations, which is an indicator of cultural affiliation relationships, is what we call the Andean imprint in the basin of Río Bueno or Wenuleufu.

Keywords: Williche, Andeanness, worldview, co-tradition.

Recibido: 16.12.14. Aceptado: 03.06.13.

W

ENULEUFU ${ }^{1}$, 'el río de arriba', es el nombre con el que denominan en mapudungun al río Bueno, principal curso fluvial de la Provincia del Ranco, situado al sur de la Región de Los Ríos. Su topónimo tiene connotaciones místicas, pues en la escatología mapuche los ríos son una de las vías por donde los espíritus transitan hacia la otra vida. Wenuleufu es, igualmente, el etnónimo de la Vía Láctea, el río de las estrellas, que refleja en el firmamento el viaje de las almas. Esta recurrencia entre la topología y la cosmografía es común al espacio central andino, donde los elementos del plano terrestre encuentran su reflejo en el plano sideral. Así, el río Vilcanota, que recorre el valle sagrado de los incas, se considera la contraparte de la Vía Láctea conocida Ch'aska Mayu (Steele y Allen, 2004), que en quechua significa río de estrellas.

Frontera natural a la vez que ruta de navegación, la hoya hidrográfica del río Bueno se nutre de las aguas de los lagos Maihue, Ranco, Puyehue y Rupanco, que llegan a él a través de numerosos afluentes, constituyendo uno de los sistemas fluviales más importantes de Chile. Se estima que este espacio ha tenido ocupación humana los últimos diez mil años ${ }^{2}$ Al momento de la llegada de los españoles, éste se encontraba habitado por poblaciones sedentarias ubicadas en las riberas de lagos y ríos, que practi-

\footnotetext{
${ }^{1}$ Para escribir los términos propios del mapudungun empleamos el Alfabeto Mapuche Unificado propuesto por la Sociedad Lingüística de Chile y destacamos las palabras en cursiva. La excepción son los topónimos donde mantenemos las formas en uso en el sistema de referencias geográficas y administrativas. En mapudungun, los sustantivos y adjetivos no emplean los sufijos 's', 'es' como pluralizadores. No obstante, en el texto usamos la expresión 'kamaskos', puesto que esta forma castellanizada se encuentra en uso en el área. En el caso de las voces quechua, empleamos igualmente cursivas para señalarlas, pero mantenemos la grafía de los textos y autores donde se encuentran referidas.

${ }^{2}$ Los fechados más antiguos registrados en la cuenca del Río Bueno por la Dirección Museológica de la Universidad Austral de Chile (2012) arrojan una datación de 2000 años AP. No obstante, los antecedentes de ocupación humana del área interlacustre se remontan entre 9.500 años AP para el sitio Marifilo (Adán et al., 2004) y los 12.500 para Monteverde (Dillehay, 2004).
} 
caban una economía mixta basada en la agricultura de legumbres, cereales y tubérculos, crianza de animales, caza y recolección de productos de los bosques. Estas agrupaciones habían desarrollado una industria alfarera con rasgos estilísticos propios y presentaban prácticas de metalurgia, rasgos convergentes con el horizonte cultural andino.

En la actualidad, la población indígena en el área tiene una representatividad que oscila entre el 39,5\% de los habitantes de la comuna de Lago Ranco; 21,95\% en Río Bueno y 14,14\% en La Unión (Ministerio de Desarrollo Social, 2009). Si bien ésta se encuentra expuesta a intensos procesos de aculturación lingüística y religiosa (Moulian, 2012), en este espacio ha sobrevivido el culto ancestral a los 'Ngen Mapu', espíritus tutelares de las comunidades mapuche williche (Moulian, 2008). A ellos se los considera 'dueños del territorio', lo que se encuentra denotado por el significado literal de este etnónimo. Se trata de espíritus mediadores (Foerster y Gundermann, 1997), en tanto interceden entre los seres humanos y Dios. Unos poseen un carácter regional, como Wenteyao, al que se le rinde culto desde San Juan de la Costa hasta la cordillera de la Región de los Ríos; otros tienen una presencia zonal, como Kintuante de la comuna de Río Bueno; algunos, en tanto, son espíritus tutelares locales, como Juanico de la comunidad de Nolguehue. Sus nombres son significantes de poder y objeto de devoción.

En las palabras de José Leonardo Cuante Loncomán ${ }^{3}$, último ñizol longko de Lago Ranco:

El abuelito Wenteyao para nosotros, como pueblo y como cultura, [es] la religiosidad que está ahí en Pucatrihue. Para nosotros es un hombre como todos, pero está en el mar, y a Él le pedimos todo, que interceda ante Dios por nosotros. Él nos da el agua, nos da el aire, nos da la sabiduría, nos da todo lo que queramos, el aire para respirar, nos da el aire para poder hablar. Y está en esa casa de piedra. Ahí es su casa. Ahí está para recibir a quien quiera, pero con la fe que tiene cada hombre o mujer. Y el que no va con fe, no lo deja entrar o si entra no lo deja salir. Lo

\footnotetext{
${ }^{3}$ José Leonardo Cuante Loncomán (1935-2008) nos acogió y brindó posibilidades de realizar un trabajo etnográfico en el lepün de Pitriuco el año 1998. Desde entonces y hasta su muerte el 2008 mantuvimos con su persona una estrecha relación de amistad y colaboración. A su generosidad y buena disposición debemos las posibilidades de realizar nuestra investigación en el área del Wenuleufu y parte importante de los conocimientos etnográficos que aquí exponemos. Agradecemos, igualmente, a Elías Millahueque y Juan Albarrán, mayores del lepün de Nolguehue, y a Roberto Gómez Antiñir y Andrés Malpu, autoridades rituales de la rogativa williche de Maihue Carimallín, la posibilidad de hacer trabajo de campo en sus comunidades y acompañarlos a la morada de sus espíritus tutelares.
} 
deja ahí hasta que baja la marea, porque la mar es muy celosa. Si vamos con mala fe, embravece el mar y no nos deja salir hasta cuando Él quiera. Por eso hay que tener un gran cuidado para ir.

Emplazadas en diversos accidentes geográficos, las moradas de los Ngen Mapu son hitos principales de un paisaje cultural que se muestra complejo y orgánico, sobreviviente como un relictus cultural, en el marco de los procesos de transformación social. Ellas son puntos de peregrinaje y motivo de interdicciones. En Pitriuco, Nolguehue y Maihue Carimallín, comunidades del sector lelfunche (de los llanos) del Wenuleufu, quienes mantienen viva su memoria se conocen como 'kamaskos'. Con este término se identifica a los miembros de las congregaciones rituales que organizan y sustentan las rogativas en las que se invoca a estos espíritus tutelares para propiciar el bienestar común.

Los 'kamaskos' son los depositarios de la religiosidad ancestral mapuche williche, pero la voz que los designa no proviene del mapudungun, sino es de origen quechua. Las prácticas y representaciones características del ciclo ritual que ellos preservan muestran numerosos rasgos panandinos, cuya recurrencia es un indicador de relaciones de filiación cultural que en este artículo procuramos relevar.

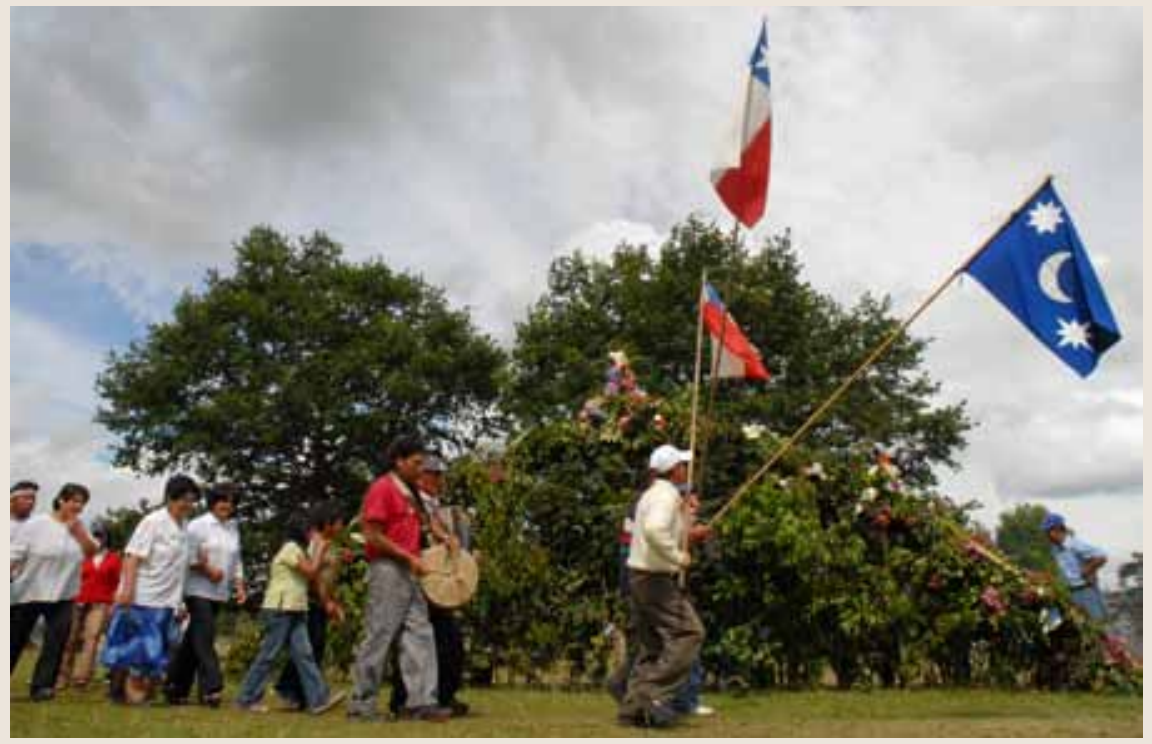

Kamaskos de la congregación ritual de Maihue Carimallín durante la rogativa comunitaria. 
El mundo andino es un mosaico de pueblos y culturas, que pese a su diversidad presentan un conjunto de rasgos comunes, que se encuentran difundidos a través del espacio cordillerano y se mantienen en el tiempo. Estos rasgos persistentes y recurrentes son los que permiten postular la existencia de una 'cultura andina' (Ossio, 2006). Esta diversidad en las formas expresivas y patrones de adaptación está, igualmente, presente en la cultura mapuche, donde encontramos manifestaciones institucionales diferenciadas en el espacio de pocos kilómetros. No obstante, bajo sus particularidades formales es posible distinguir en ellas contenidos y una lógica subyacente común que permiten reconocerlas como variantes expresivas de la misma matriz cultural. Debe tenerse en cuenta que los pueblos amerindios presentan tipos de culturas gramaticalizadas (Lotman y Escuela de Tartu, 1979). Ellas disponen de repertorios simbólicos y principios generales de producción de enunciados que generan series de transformaciones en las formas textuales, con notorias diferencias de superficie, pero con estructuras profundas compartidas. Esto es también lo que sucede en la relación de la cultura mapuche con el horizonte cultural andino.

El método que empleamos para poner en evidencia las relaciones interculturales es el estudio semiótico comparativo de tramas simbólicas que expresen paradigmas cosmovisionarios propios del espacio centro y sur andino. Por esta vía proponemos contrastar el trabajo modelizante de los sistemas culturales, deslindando las recurrencias formales explicables desde las teorías naturalistas. Es relevante tener en cuenta, al respecto, que el investigador de los sistemas simbólicos debe enfrentar la doble capacidad de predicación de sus datos, que a la vez le informan de contenidos arquetípicos de la conciencia (Jung, 1994) y de su despliegue histórico en realizaciones culturales singulares (Turner, 1999). En esta perspectiva, las representaciones simbólicas se muestran 'doblevinculares' pues ponen en escena estructuras antropológicas universales (Boyer, 1994) concretizadas en procesos sociosemióticos específicos (Geertz, 2000). Ello plantea la necesidad de precaverse de establecer presuntas relaciones históricas en virtud del hallazgo de similitudes en las formas expresivas. Para deslindar los planos y emplear los datos concurrentes en función del estudio de las relaciones interculturales temporal y espacialmente situadas, es necesario pasar desde el análisis de los arquetipos y símbolos naturales al estudio semiótico contrastante de las tramas simbólicas y paradigmas cosmovisonarios. Dicho de otro modo, es necesario someter a comparación unidades de información 
de mayor nivel de complejidad, que expongan el trabajo modelizante de los sistemas culturales que producen metamodelos cosmovisionarios (López, 1995). Es el método que aquí procuramos poner en práctica.

Nuestra experiencia de investigación etnográfica en el área del Río Bueno o Wenuleufu tiene una data de quince años y se ha plasmado en productos de investigación de diferentes formatos y soportes (Moulian y Valdés, 2002; Moulian, 2005; Moulian et al., 2008; Moulian, 2012). El punto de arranque de la investigación que hoy nos ocupa fue la toma de conciencia de la importancia de los quechuismos en el lenguaje sagrado mapuche williche (Moulian, 2008, 2009). El año 2008 comenzamos a viajar a Lima con el propósito de ahondar en el significado de estas voces, entre las que destaca 'kamasko'. En este ejercicio constatamos numerosos paralelismos entre los sistemas cosmovisionarios centro y sur andinos. Ellos se exponen de modo ejemplar en el culto a los Ngen Mapu que mantienen los kamaskos del Wenuleufu. El término 'kamasko' corresponde a uno de estos préstamos lingüísticos provenientes del quechua. Este etnónimo designa a una institución central en términos religiosos e identitarios en nuestra área de estudio, pero no se encuentra consignado en los diccionarios del mapudungun (Augusta, 1992; Cañas, 1911; Febres, 1975; Havestadt, 1777; Valdivia, 1887), porque no procede de él. Su origen es quechua. En esta lengua 'camasca' (fonológicamente $/ \mathrm{kamasqa} /$ ) designa a las personas que se benefician de una 'huaca' o entidad sagrada a la que veneran (Taylor, 2000). Es lo que sucede con los 'kamaskos' del Wenuleufu que le rinden culto comunitario a diversos espíritus que tienen alcance regional, zonal o local. El correlato lingüístico expresa un paralelismo cultural.

La noción quechua de 'camasca' deriva de la raíz semántica 'cama', cuyo significado preferente es 'animar'. Ésta conforma una serie de términos religiosos que refieren al poder vitalizante, que en la perspectiva andina anima a animales, plantas, personas y cosas y que le da sus cualidades distintivas, es decir, su identidad. Este principio creador recibe el nombre de 'camaquen'. Las entidades que lo portan se denominan 'camac'. Los lugares donde se manifiesta son las 'huacas', término que ha devenido en significante de lo sagrado y se aplica a espacios o elementos naturales (piedras, manantiales, cuevas, cerros), ídolos, templos, personas y ancestros poseedores de este poder. Los camasca son quienes a través del culto a las huacas obtienen camaquen, fuerza vital que afirma sus cualidades y especifican su identidad. Lo mismo sucede entre los kamaskos del Wenuleufu que invocan a los Ngen Mapu para recibir de ellos 'newen', como se denomina en mapudungun la fuerza vital. Así lo expresa el siguiente fragmento de oración enunciado por 
el capitán de la rogativa de Maihue Carimallín, Juan Andrés Malpu: "Defiende a tu pueblo señor Kintuante, no nos dejes caer. Usted nos da newen Señor, por eso nosotros estamos aquí. Queremos que nos dé ánimo para hacer nuestra rogativa. Danos valor, poderoso".

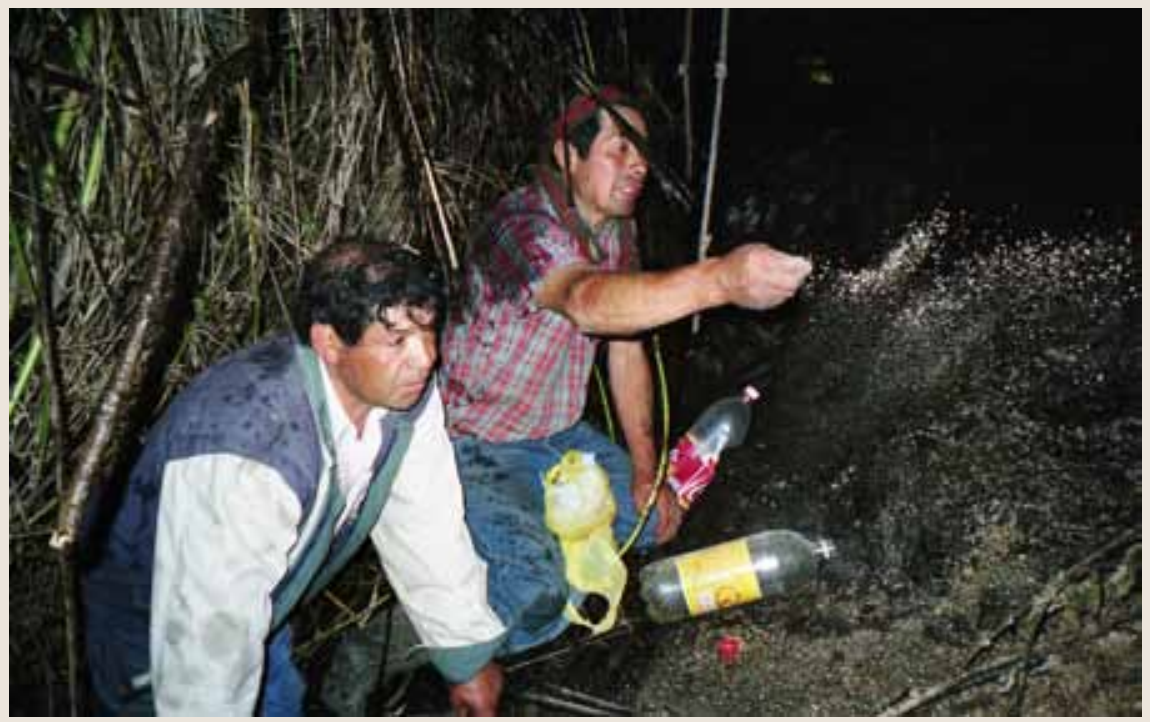

Kamaskos visitando la casa del Ngen Mapu 'Kintuante', ubicada en un renü.

\section{VÍAS DE ANCESTRALIZACÓN}

Los Ngen Mapu o espíritus tutelares mapuche williche constituyen un caso particular de ancestralización (Kaulicke, 2000), es decir, de transformación de las personas en espíritus ancestrales a los que se les rinde culto. Si bien este tópico no es exclusivo del mundo andino, aquí se presenta con rasgos que se pueden considerar marcadores de la andinidad. En nuestra área de estudio, la ancestralización se encuentra operacionalmente ligada a las características del paisaje sagrado, que se muestra poroso, vitalizado, abierto a otras dimensiones de realidad. La geografía es aquí un espacio de proyección y concreción de los modelos cosmográficos, que en el caso mapuche concibe el universo con una estructura vertical, con tres niveles fundamentales (Wenu Mapu, la tierra de arriba; Nag Mapu, la tierra de aquí, y Minche Mapu, la tierra de abajo), unidos circularmente. Cuevas, humedales, 
manantiales, ojos de agua, ríos, volcanes, especies vegetales y fenómenos atmosféricos son considerados vasos comunicantes entre estos mundos; las piedras se conciben como formas de transformación de sus fuerzas. Se trata de "mundos permeables y espacios peligros", dice Cruz (2006) respecto al espacio centro-andino, donde los etnomodelos del universo encuentran sus paralelos. Quechuas y aymaras comparten cosmografías que presentan una estructuración vertical con niveles interconectados: Hanan Pacha, Kay Pacha, Uku Pacha (en el caso quechua); Alax Pacha, Aka Pacha, Manka Pacha (en el caso aymara). Montañas, cuevas, manantiales, lagos, rayos, arcoíris se encuentran entre las instancias que comunican estos mundos, cuyas interacciones muestran una fenomenología común.

En el espacio ancestral del Wenuleufu, los Ngen Mapu son personas capturadas por este paisaje. Ellas han devenido en espíritus tutelares tras haber entrado en portales interdimensionales. Esto los convierte en seres liminares, que viven en el territorio de las comunidades, pero pertenecen al Wenu Mapu. Es el caso de Juanico, 'Ngen' de la comunidad de Nolguehue; Kintuante, de Maihue Carimallín; y Wenteyao, situado en Pucatrihue, San Juan de la Costa ${ }^{4}$. Todos ellos desaparecieron sin dejar rastros. Su destino ha sido develado por la visión de las o los machi encomendados de buscarlos o la entrega de mensajes espirituales por parte de los propios protagonistas. Los dos primeros entraron en cuevas denominadas renü, que si bien son medios de acceso a la Minche Mapu (mundo de abajo), se consideran vías que conducen al Wenu Mapu. La caracterización fenoménica de estos espacios se expresa en la etimología del término renü, compuesto por las raíces léxicas ' $r e$ ' (puro, auténtico) y nü (tomar, coger, agarrar), que se puede traducir como 'prendado por lo puro'. Es también lo que le sucede a Wenteyao, quien se enamora de una sirena (sumpall) y se queda a vivir en las rocas de Pucatrihue. Su ruka es igualmente una cueva. Una variante en este proceso de ancestralización es la litificación, la transformación de las personas en piedras. Así le ocurre a Mankian ${ }^{5}$, longko o cacique de Mariquina, quien

\footnotetext{
${ }^{4}$ Si bien la morada de Wenteyao se sitúa al sur de la cuenca del Wenuleufu, a él se le rinde culto dentro de este espacio, en comunidades como Pitriuco, ubicada en las proximidades del Lago Ranco y Marriamo, de la comuna de Río Bueno.

${ }^{5}$ La roca que representa a este Ngen Mapu se ubica en la Punta Nigue, en la desembocadura del río Toltén. No corresponde, por lo tanto, a la cuenca del Río Bueno. No obstante, el conocimiento de las características de su proceso de ancestralización es relevante para nuestra área de estudio, donde se encuentran, igualmente, espíritus litificados. Es el caso de Kurralwe (el espíritu de la piedra), en la comunidad homónima, de cuyo culto sólo quedan reminiscencias, y Santo Chaway, que se ha incorporado a la religiosidad popular de la zona de Hueicolla, en la costa de La Unión.
} 
se convierte en roca por burlarse de los poderes de un arroyo (Alonqueo, 1989). Cada una de estas vías de ancestralización (captura por espacios sagrados, enamoramiento de seres espirituales, litomormofosis) tiene sus correlatos en el mundo central andino, como se puede ver en el Manuscrito de los Ritos y Tradiciones de Huarochiri (Taylor, 1987).

Uno de los rasgos de la fenomenología de los Ngen Mapu del Wenuleufu es su sociabilidad, lo que igualmente se registra en el libro de Huarochiri. Kintuante vive al lado de su hermano mellizo Kilenwentru en la ribera del río Pilmaiquén, nos dice el capitán de la rogativa de Maihue Carimallín, Juan Andrés Malpu. La madre de ambos, María Antonia, tiene su morada en el Lago Puyehue, donde nace el referido curso fluvial. En el cerro Trengtreng de la isla Huapi, Lago Ranco, cohabitan Tripayantü y la ñuke Nillifma que conforman pareja. Wenteyao tiene entre sus colaboradores a los sargentos Mindalican y Pindalican (Gissi, 1997). Su hija es igualmente una figura ancestralizada. En San Juan de la Costa consideran que estos espíritus realizan grandes reuniones denominadas 'putrankawin'. Si bien los Ngen Mapu velan por los intereses de los miembros de las comunidades, son celosos de sus atenciones rituales y castigadores de las faltas y transgresiones. De allí el cuidado que deben mantener los kamaskos.

\section{PAISAJES ORGÁNICOS}

Previo a la realización de las rogativas, los miembros de las congregaciones rituales del Wenuleufu emprenden un viaje a la morada de los espíritus tutelares. Van a pedir autorización de los Ngen para realizar sus ceremonias e invitarlos a participar de ellas. Este gesto es un rasgo común a la religiosidad andina. Así, por ejemplo, la fiesta de la champería o limpia de canales en San Pedro de Casta, en las sierras de la región de Huarochiri (Lima), se inicia con la visita a Pariapunku, dueño del agua, quien vive en una cueva. La peregrinación hacia las huacas es un gesto común en el mundo andino. Una de las más notorias es la romería al Señor del Qoyllur rit’i ubicado en el nevado Sinakara, perteneciente a la cadena montañosa del Ausangate, Apu principal de Cusco (Flores Ochoa, 1990), considerado una entidad espiritual; una práctica que se realiza desde el período prehispánico. Aunque a escala, este gesto se repite en las comunidades andinas, donde se encuentra ritualizado el viaje hacia los espacios de manifestación del poder, un patrón concurrente en la cultura mapuche williche. En la isla Huapi del Lago Ranco, por ejemplo, los comuneros visitan el cerro Trengtreng, punto más 
alto de la isla, donde habita Tripayantü y Nillifma. Los kamaskos de Nolguehue y Maihue se dirigen hacia los renü ubicados en las inmediaciones de sus comunidades donde viven Kintuante y Juanico. Los miembros de la congregación de Pitriuco viajan ciento ochenta kilómetros hasta la casa del Abuelito Wenteyao en la roca de Pucatrihue. Su desplazamiento enfatiza la importancia de la topografía sagrada como espacio de la manifestación de las fuerzas y entidades espirituales que, pese a ser ubicuas, se encuentran localizadas, emplazadas materialmente en el paisaje.



Peregrinaje de los kamaskos de la comunidad de Pitriuco a la morada del Abuelito Wenteyao en las rocas de Pucatrihue.

Los sitios donde residen los Ngen Mapu son uno de los componentes de los paisajes simbólicos que muestran un carácter orgánico, en tanto los espacios que los conforman se encuentran estructural y funcionalmente interrelacionados. Los complejos sagrados de Maihue y Carimallín, en los márgenes del río Pilmaiquén y Nolguehue, sobre el río Muticao, ilustran de manera ejemplar esta articulación, sirviéndonos de modelos para el análisis. En ellos encontramos un patrón común de organización del espacio cúltico que integra la morada de los espíritus tutelares, los cementerios comunitarios y las canchas de rogativa. Los lepün o ngillatun williche, ritual de 
solicitud y acción de gracias que anualmente organizan los kamaskos muestran la vigencia de estos paisajes sagrados. Estas ceremonias son instancias de comunicación con Dios e interacción con los Ngen Mapu, antepasados y fuerzas de la naturaleza. Las pautas de acción y organización simbólica del espacio ceremonial comprometen representaciones teológicas, pneumatológicas, escatológicas, naturalistas y cosmológicas y permiten visualizar las recurrencias en las disposiciones y prácticas rituales asociados a estos dominios. Los paradigmas cosmovisionarios contenidos en sus tramas simbólicas dejan en evidencia la impronta andina.

Lo vemos, por ejemplo, en el campo de la escatología. En el área del Wenuleufu los cementerios indígenas cumplen con una doble propiedad: se sitúan en la proximidad de los cursos de agua y en explanadas desde donde se domina la visión de los volcanes del área. Este patrón de emplazamiento se registra desde el período Pitrén (Alvarado y Mera, 2004). En él se expresan las principales variantes escatológicas mapuche: el desplazamiento de las almas a través de los ríos siguiendo los pasos del sol (Sanfuentes, 1925) o su entrada por los volcanes (Calvo, 1987). Estas representaciones del camino que emprenden los muertos hacia la otra vida tienen un carácter panandino. El cruce de ríos en el lomo de perros negros, el viaje a través del mar sobre lobos marinos, el movimiento en dirección poniente son tópicos presentes en el espacio moche (Millones, 2010), laymi (Harris, 1983) uro chipaya (Acosta, 1998) y aymara (Motta, 2011). La variante del viaje a la montaña se encuentra en la población quechua de Arequipa (Valderrama y Escalante, 1980) y Cusco (Robin, 2005). Al respecto, Gutiérrez (1938, p. 129) en Huilla-cuyun-ches (Leyendas de la gente de Cuyo), registra el desplazamiento meridional de estas representaciones escatológicas al sur de Mendoza:

Existía cerca de las huakas de la costa del Chadileuvu (Río Salado) el Upa Marka, lugar del silencio y del arrepentimiento, que se ubica indistintamente en la isla de una laguna (Llancanelo, Diamante, etc.) o en el hueco de un cráter de un volcán, a la orilla de una laguna (Laguna de la Niña Encantada).

El patrón de emplazamiento de los cementerios (eltun) de Maihue y Nolguehue se establece en relación con estos caminos que conducen hacia la otra vida, los que deben tenerse a la vista para el desarrollo eficaz de las acciones rituales. Éstos se sitúan próximos a los cursos de agua en puntos desde donde se domina el volcán Osorno. Las moradas de los Ngen Mapu se encuentran, igualmente, en renü situados en sus proximidades, que consti- 
tuyen puertas dimensionales, donde el paso de los espíritus al Wenu Mapu se produce de manera directa. En la comunidad de Maihue se ubica en una quebrada en los márgenes del río Pilmaiquén, uno de los principales tributarios del río Bueno. En Nolguehue se encuentra en el cauce de una vertiente que recoge las aguas de la comunidad y desemboca en el río $\mathrm{Mu}$ ticao, tributario del anterior.



Paisaje escatológico en Maihue: vista del cajón del río Pilmaiquén y del volcán Osorno.

El principio de estructuración dual marca la configuración de estos espacios. En Maihue, se compone de dos cuevas colindantes labradas por la acción del agua que se filtra por las paredes. En ellas residen Kintuante, espíritu tutelar, y su mellizo Kilenwentru, médico espiritual. En Nolguehue, el sitio está marcado por desniveles sucesivos que producen caídas de agua. El protocolo de la acción ritual que desarrollan los kamaskos supone la realización de oraciones y entrega de ofrendas en estos dos puntos. La paridad en la realización de las acciones constituye aquí una pauta generalizada, que es recurrente en el mundo andino. Para los mapuche lo impar aparece como incompleto, imperfecto o mal hecho (Briones de Lanata y Olivera, 1985). 


\section{SÍMBOLOS NATURALES DE MEDIACIÓN}

Las rogativas que anualmente organizan los kamaskos son instancias de mediación ritual destinadas a efectuar solicitudes y pagos dirigidos a Dios por medio de la intercesión de los Ngen Mapu. En ellas se sintetizan las diversas dimensiones del universo mapuche, cuyas entidades interactúan en la ceremonia. Ellas presuponen la concurrencia de los espíritus de los antiguos kamaskos, ya fallecidos, tanto como la relación con los elementos y las fuerzas de la naturaleza. Para realizar esta comunicación, en Nolguehue y Maihue los kamaskos tienden simbólicamente un arco que conecta los distintos niveles del mundo. Éste se elabora de varas de avellano cubiertas por ramas y flores que le añaden diversidad de colores y tiene la función de rewe, instrumento sagrado y punto de interacción entre hombres y espíritus. Los kamaskos dicen que es una representación del arcoíris o relmu, el camino al cielo. Este fenómeno atmosférico se considera un portal que conduce hacia el Wenu Mapu, con una función similar a los renü. Así lo hemos registrado en un nütram recogido en la comunidad de Alepué, donde el arcoíris que se levanta en una caída de agua o trayenco, constituye una vía por el cual se produce la sustracción de las almas.

En el área del Wenuleufu, el simbolismo del arco aparece igualmente en el marco de rituales funerarios con una función similar: favorecer la partida del alwe o alma. Su uso también se encuentra registrado en contextos chamánicos como umbral que marca el kuymün o entrada del espíritu en los machi (Flury, 1944; Bacigalupo, 2007). En forma de rewe también se emplea en la rogativa de Tringlo (Lago Ranco) y, antaño, en Pitriuco (Lago Ranco), lo que informa de un patrón ritual más extenso. Una asociación simbólica interesante se presenta en la comunidad de Maihue, donde el arco se encuentra coronado con una cruz de flores denominada 'cruceta' que ocupa la posición central del mismo. Esta relación entre el arco y la cruz se registra, igualmente, en el arte rupestre del sitio Peumayén 2, ubicado en el paralelo 42, en la vertiente oriental de los Andes (Podestá et al., 2009). Dos figuras humanas con manos alzadas se sitúan bajo esta imagen, exponiendo una expresión ritual que se mantiene hasta el presente entre los williche. Ello resulta relevante, porque la similitud y proximidad geográfica con el ngillatue de Maihue sugiere relaciones de filiación cultural; en tanto, la datación contextual del sitio con actividad pictórica desde hace 1.200 años AP muestra la antigüedad de este patrón expresivo. 




Sitio Peumayén 2 y la representación del arco del cielo.

En el sitio Peumayén 2, el arco asociado a la cruz se encuentra flanqueado por otros simples, lo que indica un dualismo en sus manifestaciones: el arco del día (arcoíris) y el de la noche (vía láctea). Cuando la constelación de la Cruz del Sur alcanza su posición cenital, los primeros días de mayo, esta última exhibe la misma relación figurativa presente en la pintura de Peumayén y en el rewe de Maihue. En la mitología andina el arcoíris y la vía láctea se encuentran representados por la serpiente de dos cabezas o anfisbena, animal que simboliza la conexión entre los diversos estratos del mundo y que desde el formativo andino se lo figura icónicamente en forma de arco. Éste es un símbolo de mediación extensamente empleado en los Andes: se encuentra desde la cultura moche, en los frisos de la Huaca de la Luna y Huaca Señora de Cao, hasta la cultura inca, en los queros y el cosmograma de Coricancha. Nuestro registro etnográfico ha detectado su vigencia en el mundo aymara como símbolo del ayni (encuentro), también como portal para el paso de las almas para el 2 de noviembre, conocido como la fiesta de ayamarca, día en el que regresan las ánimas. Entre cayawayas y likanantay o atacameños presenta igualmente esta función de umbral para el día de los difuntos.

En las rogativas williche de Maihue y Nolguehue los arcos son las puertas por las que llegan los espíritus de los külche (kiluches, en la expresión castellanizada), seres del país de las entrañas, como se denomina a los difuntos. 
Apenas los kamaskos comienzan a levantar sus rewe perciben la llegada de los vientos, indicadores de la presencia de los espíritus. Así nos testimonió Juan Albarrán, mayor de la rogativa de Nolguehue: "Cuando enterramos las varas de avellano se sienten los espíritus que vienen de la costa en forma de aire". Bajo el arco dejarán las ramas que representan simbólicamente a los Ngen Mapu, las que son sometidas a especiales cuidados rituales. El rewe será el centro de las acciones rituales consistentes en la ofrenda de alimentos, libaciones con chicha, sahumerios, sacrificios de animales, asperges con sangre y bailes. La estructura profunda de este tipo ritual es equivalente a la recepción de los difuntos en torno a arcos de mesas o altares familiares, vigentes entre aymaras, kallawayas y likanatay. Aunque con funciones diferenciadas, la acción performativa de ambos rituales se dirige al encuentro entre los miembros de las congregaciones y sus ancestros espirituales. Sus tramas litúrgicas están marcadas por similares símbolos de mediación y mecanismos de ritualización, donde predomina el sacrificio de alimentos y las expresiones musicales.

\section{HORIZONTE CULTURAL PANANDINO}

Entre los mapuche williche predomina en la actualidad una concepción teológica monoteísta, incorporada a través de la evangelización católica. Ésta se representa en la figura de Chaw Dios (Dios Padre) al que se dirigen sus oraciones. No obstante, un sustrato anterior de carácter naturalista y politeísta se mantiene codificado en diversos elementos significantes de la rogativa. Ellos se expresan de manera explícita en el discurso cúltico de San Juan de la Costa que implora: 'leuketumayen Chaw Antül leuketumayen Ñuke Ale' (Acuérdate de nosotros Padre Sol/ Acuérdate de nosotros Madre Luna). La apelación dual expresa la idea de familia divina vigente en otras áreas mapuche, constituida por Wenu Mapu Fucha (El mayor de cielo), Wenu Mapu Kushe (la anciana celestial), Wenu Mapu Wentru (el joven celeste), Wenu Мари Ülcha (la doncella del cielo). Estas entidades se asocian figurativamente al Sol, la Luna, el Lucero de Anochecer y el Lucero del Amanecer, una configuración iconográfica que se repite en el famoso cosmograma inca del templo Coricancha (Santa Cruz Pachacuti, 1995). Las wipala o banderas williche de Nolguehue y Maihue resaltan el vínculo entre la luna naciente y los luceros. La orientación del espacio ritual y la fecha del calendario señalan la preeminencia simbólica del sol. 




Rewe de arco en la comunidad de Litrán.

En Maihue la realización de las rogativas coincide con el solsticio de verano, lo que también ocurría antiguamente en Nolguehue. La onomástica de los Ngen Mapu de estas comunidades expresa, también, un simbolismo de carácter astral. Kintuante significa 'el que busca el sol o la claridad'; el nombre de Juanico alude al agua donde los kamaskos se bañaban en la fiesta de San Juan que enmascara al Wetripanantu, como denominan al año nuevo mapuche, correspondiente al solsticio de invierno. En ambos sitios, el 'lepuntue' o lugar de rogativa, muestra una orientación hacia el levante, cuestión que es recurrente en las canchas rituales andinas, orientadas o abiertas hacia el levante. Este patrón es una constante en la organización del espacio mapuche, que se verifica en la disposición de las tumbas en los cementerios, en la orientación que asumen los oficiantes en rituales chamánicos, la estructuración de los espacios habitacionales, con las puertas de las ruka y la disposición de las camas mirando hacia el este. En el marco de la cultura mapuche los movimientos rituales y protocolares siguen el sentido de la rotación y traslación de la tierra en torno al sol, en un transcurso antihorario, que va de derecha a izquierda. Estas disposiciones espaciales son coincidentes con las que ordenan el mundo aymara (Motta, 2011) y de pastores quechuas (Pino, 2004). En el registro arqueológico de las mismas nos remiten hasta el período formativo, expuesto, por ejemplo en los centros ceremoniales de Chavín o Pukara. 
Los astros, el arco y las cuevas son símbolos naturales, que exhiben relaciones significativas sobre bases indiciales y principios analógicos y metafóricos. Se trata, por lo tanto, de elementos potencialmente recurrentes, pero igual complejos y ambivalentes, abiertos a variantes en sus representaciones e interpretación y con distintas posibilidades de combinación. La lectura de cada una de ellos implica connotaciones, presupuestos y plantea puntos de articulación, en los que se expresa la urdiembre cultural. En este nivel es donde advertimos los paralelismos: en las asociaciones entre estos elementos simbólicos con su carga de connotaciones, en tramas simbólicas que expresan paradigmas cosmovisionarios, es decir, la actividad modelizante de la cultura.

Lo que denominamos 'impronta andina' es la recurrencia de componentes de los paradigmas cosmovisionarios propios del espacio central andino entre los kamaskos del Wenuleufu, lo que consideramos un indicador de relaciones de filiación cultural. Entre los elementos correlacionados se cuentan las formas de ancestralización, las nociones escatológicas, una concepción orgánica e intersticial de los paisajes culturales, la representación de los elementos de la naturaleza como entidades numinosas o significantes de las mismas, la estructuración vertical y articulación circular de los modelos cosmológicos y los patrones litúrgicos de mediación. Se puede constatar la existencia de paralelismos formales en diversos sistemas expresivos, desde el lenguaje hasta las modalidades de acción performativa; la presencia de principios de organización cognitiva comunes y modelos paradigmáticos compartidos. Al menos, parte de estas correspondencias se pueden rastrear desde el período formativo (Pitrén), lo que indica relaciones de larga data. El carácter sistemático de las mismas, que se expresa a niveles de las tramas simbólicas y articulan diversas dimensiones de los sistemas religiosos y cosmovisionarios, informa de un horizonte cultural panandino, en el que se inscriben las prácticas y representaciones de los kamaskos del Wenuleufu.

\section{REFERENCIAS}

Acosta, O. (1998). "La muerte en el contexto uru: caso chipara". Eco Andino. 5, 7-40.

Adán, L.; Mera, R.; Becerra, M. y Godoy, M. (2004). “Ocupación arcaica en territorios boscosos y lacustres de la región precordillerana andina del centro-sur de Chile. El sitio Marifilo 1 de la Comunidad de Pocura". Revista Chungara, Volumen especial, 1121-1136. 
Alonqueo, M. (1989). “Mankian”. En Hidalgo, J. (ed.), Culturas de Chile. Etnografía sociedades indígenas contemporáneas y su ideología (pp. 232-233). Santiago: Editorial Andrés Bello.

Alvarado, M. y Mera, R. (2004). "Estética del paisaje y reconstrucción arqueológica. El caso de la región de Calafquén”. Chungara, Volumen especial, 559568.

Augusta, F. (1992). Diccionario mapuche-español y español-mapuche. Santiago: Ediciones Séneca.

Bacigalupo, M. (2007). Shaman of the foye tree. Gender power and healing among Chilean Mapuche. University of Texas Press. Austin.

Boyer, P. (1994). The naturalness of religious ideas: a cognitive theory of religion. University of California Press. Berkeley.

Briones de Lanata, C. y Olivera, M. Á. (1985). "Che Kimin. Un abordaje a la cosmo-lógica mapuche”. Runa, Vol. XV, pp. 43-81.

Calvo, M. (1987). Secretos y tradiciones mapuches. Editorial Andrés Bello.

Cañas Pinochet, A. (1911). Estudios de lengua veliche. Santiago: Imprenta Barcelona, Santiago.

Cruz, P. (2006). "Mundos permeables y espacios peligrosos. Consideraciones sobre punkus y qaqas en el paisaje altoandino de Potosí, Bolivia". Boletín del Museo Chileno de Arte Precolombino, 11(2), 35-50.

Dillehay, T. (2004). Monteverde: un asentamiento humano del pleistoceno tardio en el sur de Chile. Santiago: Lom.

Dirección Museología de la Universidad Austral de Chile (2012). Arqueología de Lago Ranco. http://www.museosaustral.cl/index.php/investigacion/proyectos/proyectos-en-curso . Consultado el 5 de febrero de 2013.

Febres, A. (1975) [1764]. Arte de la lengua del Reyno de Chile. Cabildo: VaduzGeorgetown.

Flores Ochoa, J. (1990). “Taytacha qoylluriti. El Cristo de la nieve resplandeciente". En Flores Ochoa, J. El Cuzco, resistencia y continuidad. Cusco: Ed. Andina.

Flury, L. (1944). Guilliches. Tradiciones, leyendas, apuntes gramaticales y vocabulario de la zona pampa araucana. Córdoba: Imprenta de la Universidad de Córdoba.

Foerster, R. y Gundermann, H. (1997). "Religiosidad mapuche contemporánea: elementos introductorios". En Hidalgo, J. et al., Culturas de Chile. Etnografía. Santiago: Ed. Andrés Bello.

Geertz, C. (2000). La interpretación de las culturas. Barcelona: Gedisa.

Gissi, N. (1997). Aproximación al conocimiento de la memoria mapuche williche de San Juan de la Costa. Tesis de Antropología. Universidad de Chile. Santiago.

Gutiérrez, J. (1938). Huilla-cuyun-ches (Leyendas de las gentes de cuyo). Buenos Aires: Talleres de la Cárcel de Encausados.

Harris, O. (1983). "Los muertos y los diablos entre los laymi de Bolivia”. Chungara, 11, 135-152. 
Havestadt, B. (1777). Chiledungu. Typis B. G. Teubneri.

Jung, C. (1994). Arquetipos e inconsciente colectivo. Barcelona: Paidós.

Kaulicke, P. (2000). Memoria y muerte en el antiguo Perú. Lima: Pontificia Universidad Católica del Perú, Fondo Editorial.

López, A. (1995). “Tras un método de estudio comparativo entre las cosmovisiones mesoamericana y andina a partir de sus metodologías”. Anales de Antropología, 32, 209-240.

Lotman, J. y Escuela de Tartu (1979). Semiótica de la cultura. Madrid: Cátedra.

Millones, L. (2010). Después de la muerte: Voces del limbo y el infierno en territorio andino. Lima: Fondo Editorial del Congreso del Perú.

Ministerio de Desarrollo Social (2009). Encuesta de Caracterización Socioeconómica Nacional (CASEN). En Reportes Estadísticos Comunales. Biblioteca del Congreso, Chile.

Motta, E. (2011). Pacha: visión andina del espacio tiempo en la perspectiva del pensamiento seminal. Tesis de doctorado en Filosofía. Universidad Nacional Mayor de San Marcos. Lima.

Moulian, R. (2005). Tiempo de lepün: una etnografía visual del ngillatun williche. Valdivia: Universidad Austral de Chile \& Programa Orígenes.

Moulian, R. (2008). 'Kamaskos y kamarricos en los andes meridionales. Préstamos lingüísticos y horizontes socioculturales de las instituciones rituales mapuche williche”. Ponencia presentada al VI Congreso Internacional de Etnohistoria. Universidad Católica del Perú, Lima. Inédita.

Moulian, R. (2009). "Quechuismos en el lenguaje sagrado mapuche williche como encrucijadas de sentido". Ponencia presentada en el XVI Congreso del Hombre y la Cultura Andina y Amazónica. Universidad Nacional Mayor de San Marcos, Lima. Inédita.

Moulian, R. (2012). Metamorfosis ritual. Desde el ngillatun al culto pentecostal. Teoría, historia y etnografía del cambio ritual en comunidades mapuche williche. Valdivia: Kutrún \& Universidad Austral de Chile.

Moulian, R. y Valdés, C. (2002). Abuelito Huenteao, mito y ritual. Documental etnográfico. Valdivia: Universidad Austral de Chile \& Fondo Nacional para el Desarrollo de la Cultura y las Artes.

Moulian, R.; Contreras, P.; Figueroa, A.; Valdés, C. (2008). Ngen Rüpu: una etnografía multimedia y trabajo de arqueología de los símbolos. Valdivia: Universidad Austral de Chile \& Fondo Nacional para el Desarrollo de la Cultura y de las Artes.

Ossio, J. (2006). “Andinidad”. Boletín del Instituto Riva Agüero, 33, 59-63.

Pino, J. L. (2004). "El ushnu inka y la organización del espacio en los principales tampus de los wamani de la sierra central del chinchaysuyu". Chungara, 36, 306-311.

Podestá, M.; Albornoz, A. M.; Vasini, A. y Tropea, E. (2009). "El sitio Peumayén 2 en el contexto del arte rupestre del bosque andino patagónico”. Comechingona Virtual, 3 (2), 117-123.

Robin, V. (2005). "Caminos a la otra vida: ritos funerarios en los andes perua- 
nos meridionales”. En Molinié, A. (ed.), Etnografías del Cuzco (pp. 47-68). Cusco: Centro Bartolomé de las Casas.

Sanfuentes, S. (1925). "Memoria sobre el estado de las misiones en esta provincia en 1846, pasada al gobierno por el Intendente de la misma don Salvador Sanfuentes". En Bauer, K. (comp.), Valdivia antes de la inmigración. Valdivia: Imprenta Borneck.

Santa Cruz Pachacuti Yamqui Salcamaygua, J. (1995). Relación de antigüedades de este reino del Perú. Lima: Fondo de Cultura Económica.

Steele, P. y Allen, C. (2004). Handbook of inca mythology. Santa Bárbara: ABCCLIO.

Taylor, G. (1987). Ritos y tradiciones de Huarochiri del siglo XVII. Lima: Instituto de Estudios Peruanos.

Taylor, G. (2000). Camac, camay y camasca. Lima: Centro de Estudios Regionales Andinos Bartolomé de las Casas \& Instituto Francés de Estudios Andinos.

Turner, V. (1999). La selva de los símbolos. Madrid: Siglo XXI.

Valderrama, R. y Escalante, C. (1980). "Apu Qorpuna (visión del mundo de los muertos en la comunidad de Awkinmarka)". Debates de Antropología, 5, 233-269.

Valdivia, L. de (1887). Arte y gramática general de la lengua que corre en todo el Reyno de Chile, con un vocabulario y confesionarios de la Compañía de Jesús en la provincia del Pirú. Leipzig: Ausgabe von Platzmann. 